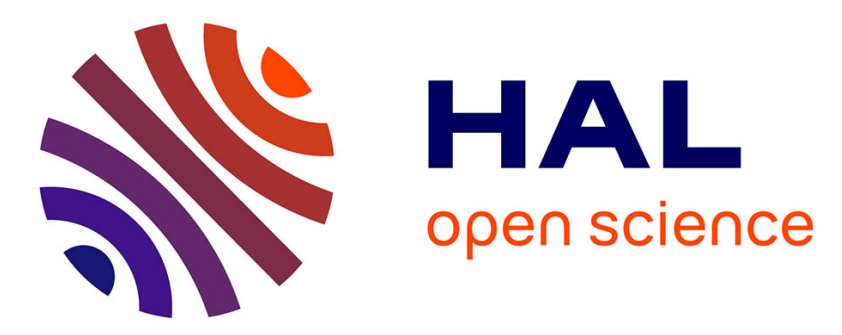

\title{
Feedforward and IMC-feedback control of a nonlinear 2-DOF piezoactuator dedicated to automated micropositioning tasks.
}

\author{
Micky Rakotondrabe, Joël Agnus, Philippe Lutz
}

\section{- To cite this version:}

Micky Rakotondrabe, Joël Agnus, Philippe Lutz. Feedforward and IMC-feedback control of a nonlinear 2-DOF piezoactuator dedicated to automated micropositioning tasks.. IEEE International Conference on Automation Science and Engineering, CASE'11., Aug 2011, Trieste, Italy. pp.393-398. hal-00709779

\section{HAL Id: hal-00709779 \\ https://hal.science/hal-00709779}

Submitted on 19 Jun 2012

HAL is a multi-disciplinary open access archive for the deposit and dissemination of scientific research documents, whether they are published or not. The documents may come from teaching and research institutions in France or abroad, or from public or private research centers.
L'archive ouverte pluridisciplinaire HAL, est destinée au dépôt et à la diffusion de documents scientifiques de niveau recherche, publiés ou non, émanant des établissements d'enseignement et de recherche français ou étrangers, des laboratoires publics ou privés. 


\title{
Feedforward and IMC-feedback control of a nonlinear 2-DOF piezoactuator dedicated to automated micropositioning tasks
}

\author{
Micky Rakotondrabe, Member, IEEE, Joël Agnus and Philippe Lutz Member, IEEE,
}

\begin{abstract}
This paper presents the characterization, modeling and precise control of a 2-dof piezoactuator dedicated to precise and automated micropositioning tasks. The piezoactuator is characterized by a strong hysteresis and a high coupling between the two axes making the synthesis of a controller very difficult. We therefore propose to compensate first the hysteresis (feedforward control) in order to obtain an approximate linear system. Afterwards, an internal model control (IMC) structure is applied (feedback control) to enhance the performances of the piezoactuator. The main advantage of the proposed approach is its simplicity both for computation and for implementation making it very convenient for realtime embedded systems. Finally, the experimental results demonstrate its efficiency and conveniency for precise positioning.
\end{abstract}

\section{INTRODUCTION}

Piezoelectric materials are very prized for the design and development of devices working at the micro/nanoscale. This recognition is due to the large bandwidth, high resolution and high force density that they can offer.Their applications are various: surface scanning and microforce measurement (AFM microscopy), microassembly (gripper, etc.), medical surgery in confined space, reader in hard-disk drivers, high precision positioning (micro/nanopositioning), etc. In the field of micro/nano-positioning where the required accuracy is micrometric or submicrometric, several principle of devices have been developed: stick-slip [1][2][3], inch-worm [4], ultrasonic [5] and bending microactuators [6]. In bending microactuators, the principle is that a cantilever based on two or several piezoelectric layers (piezolayers) bends when a voltage is applied to the latters. These actuators are particularly of interest in high speed tasks because it is possible to increase the bandwidth beyond the kiloHertz if they are appropriately controlled.

The development of single degrees of freedom (1-dof) bending piezoactuators has been very broad [6]-[10] They allowed the precise positioning of small objects with micrometric or submicrometric accuracy and with a settling time less than a hundred milliseconds. However, these actuators exhibit a hysteresis nonlinearity when the bending range is increased by applying higher input

FEMTO-st Institute,

UMR CNRS-6174 / UFC / ENSMM / UTBM

Automatic Control and Micro-Mechatronic Systems department (AS2M department)

25000 Besançon - France

corresponding author: mrakoton@femto-st.fr electric field. This nonlinearity undeniably makes the loss of accuracy if not controlled. In the literature, the control of nonlinear 1-dof bending piezoactuators has reached its maturation and can be classified into three categories: feedforward, feedback and feedforward-feedback control. In feedforward control, the hysteresis as well as other nonlinearities (creep) and vibration are precisely modelled. Then a kind of inverse model (called compensator) is put in cascade with the process in order to enhance its performances [11]-[13]. The main advantage is the packageability of the whole system since no sensor for feedback is required. However, as soon as the process is subjected to external disturbances or if the model used is uncertain, the performances are lost. In feedback category, the nonlinearities are approximately modelled and a linear closed-loop control based on these approximate models is synthesized ([14] and references herein). Finally, feedforward-feedback consists in first linearizing the process by using a compensator in cascade, and then applying a simple linear feedback controller. As the hysteresis is compensated, the main advantage of this approach is the possibility to make the actuator work in a very large bending range [15].

While the development and control of 1-dof bending piezoactuators are almost well established, this is not the case for 2 or multiple dof actuators. First, the development of bending piezoactuators having more than 1-dof is new [16][17]. In fact, certain recent applications such as spatial (x-y-z) micropositioning or those which combines positioning and orientation require dexterous devices and thus single dof actuators are not anymore efficient. The control of multiple dof piezoactuators is also recent. Additionally to the hysteresis of the material, the main difficulties of the controller design lie on how to account the coupling between the different axis. For that, in our previous work [18], we proposed an approach to control the 2-dof piezoactuator developed in [16]. In this approach, we first used a feedforward compensator to linearize the system. Afterwards we synthesized a $H_{\infty}$ controller in order to improve the performances of the actuator. However, the technique led to a high order controller that made it impossible for real-time embedded applications. Furthermore, the synthesis of the $H_{\infty}$ controller required to precisely identify the coupling between axis, which finally made the approach very complex. In this paper, we propose a more simple feedforwardfeedback approach to control 2-dof piezoactuators. While the hysteresis is compensated with a Prandtl-Ishlinskii 
approach, an internal model control (IMC) structure is proposed for the feedback. The main advantages relative to previous works are the simplicity of the control scheme and the low-order of the controller making it very adaptable for real-time embedded systems. Furthermore, the modeling aspect is very simplified since no specific characterization and modeling of the coupling is required. Indeed, the coupling is automatically accounted by the IMC structure.

The paper is organized as follows. In section-II we present and characterize the nonlinear 2-DOF piezoactuator to be controlled. Section-III is dedicated to the feedforward control of the hysteresis. Finally, in sectionIV, the modeling and the IMC-feedback control synthesis are detailed and the experimental results are presented.

\section{The 2-DOF PIEZEOACTUATOR}

\section{A. Presentation of the piezeoactuator}

The 2-DOF piezocantilever used in this paper has the same principle than the 2-DOF bilayered piezocantilever developed in [16] but is made of 36 piezoelectric layers instead of 2. Such high number of layers allows using low voltage to obtain the same output deflection. Indeed, as we will see in the next subsection, it is possible to reach up to $30 \mu \mathrm{m}$ of deflection with only $10 \mathrm{~V}$ of input voltage with the new multylayered cantilever against $40 \mathrm{~V}$ with the bilayered one. Fig. 1 presents a photography of the piezocantilever. The total dimensions of the active part are: $25 \mathrm{~mm} \times 1 \mathrm{~mm} \times 1 \mathrm{~mm}$. The piezocantilever has two input voltages $U_{y}$ and $U_{z}$ and can perform a deflection along $y$-axis or $z$-axis.

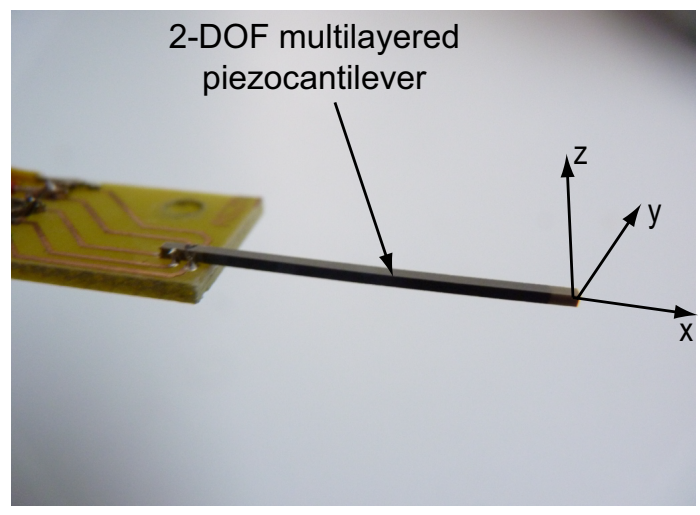

Fig. 1. The 2-DOF multilayered piezocantilever.

The whole experimental setup is composed of:

- the piezocantilever,

- two optical sensors allowing the measurement of $y$ and $z$ deflections. The sensors (from Keyence) have $10 \mathrm{~nm}$ resolution and $\approx \pm 100 \mathrm{~nm}$ accuracy,

- a computer and a dSPACE-board working in realtime with a sampling-time of $0.2 \mathrm{~ms}$. The MatlabSimulink software is used to implement the controller and to manage the different signals,
- and two synchronized high-voltage amplifiers that amplifies the control signals from the dSPACEboard.

\section{B. Characterization of the piezeoactuator}

In this part we characterize the 2-DOF piezoactuator. First we study the static part in order to evaluate the range of deflections in the two axis as well as the coupling between them. For that, we apply a sine input voltage $U_{y}$ and let $U_{z}=0 \mathrm{~V}$. While the amplitude is $10 \mathrm{~V}$ (and then $5 V$ ), the frequency is chosen to be relatively low $(f=0.1 \mathrm{~Hz})$ in order to be sure that the dynamics of the cantilever will not affect the static characteristics through the phase-lag [14]. Then, the deflections $y$ and $z$ are measured and plotted w.r.t. $U_{y}$ (see Fig. 2-a and c respectively). Afterwards, we set $U_{y}=0 \mathrm{~V}$ and apply a sine input $U_{z}$. Again the amplitude is $10 \mathrm{~V}$ (and then $5 \mathrm{~V}$ ) and the frequency is chosen to be $f=0.1 \mathrm{~Hz}$. After measuring the deflections $y$ and $z$, they are plotted w.r.t. $U_{z}$ (see Fig. 2-b and d respectively). As we can see, the direct transfers $U_{y} \rightarrow y$ and $U_{z} \rightarrow z$ are typified by hysteresis (Fig. 2-a and d respectively). Furthermore, we can see the remarkable existence of coupling transfers $U_{z} \rightarrow y$ and $U_{y} \rightarrow z$ (Fig. 2-b and c respectively). Both the hysteresis and the coupling make the piezocantilever lose accuracy. This is why they have to be minimized thanks to a feedforward and/or a feedback control.

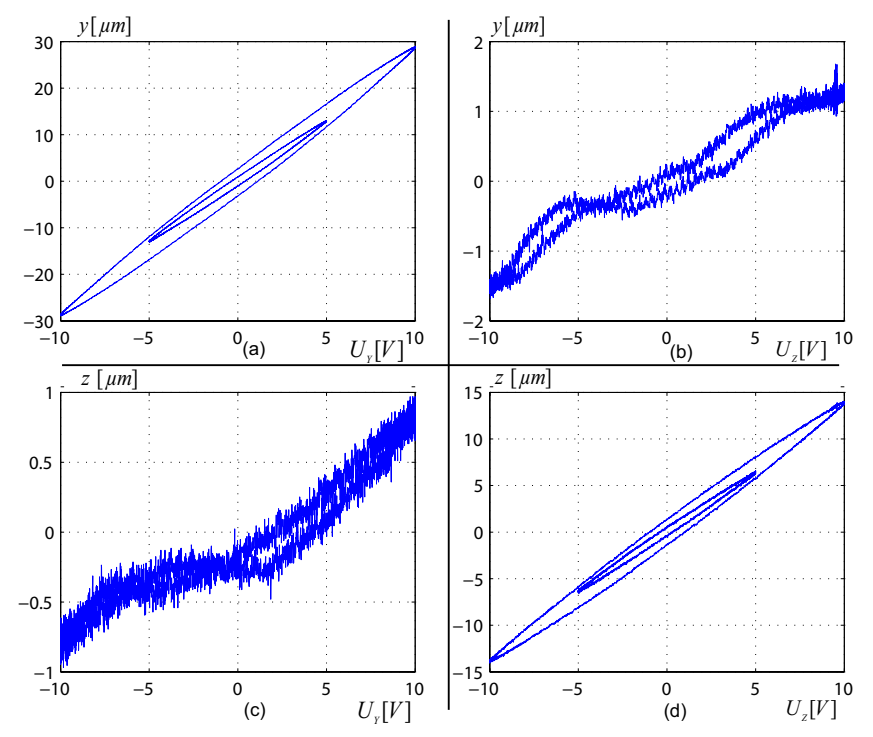

Fig. 2. Static characteristics of the piezocantilever.

We now characterize the dynamics of the cantilever. For that we use step input signals and regard the corresponding step responses. First, let us apply a step $U_{y}=$ $10 \mathrm{~V}$ and set $U_{z}=0 \mathrm{~V}$. The resulting output deflection $y$ is plotted in Fig. 2-a while that of $z$ is in Fig. 2-c. Afterwards, we set $U_{y}=0 \mathrm{~V}$ and apply a step $U_{z}=10 \mathrm{~V}$. The resulting deflection $y$ is plotted in Fig. 2-b while that of $z$ is in Fig. 2-d. Again, we confirm the presence of coupling in the axis (Fig. 2-b and c). Furthermore, we see 
that the direct step responses are very oscillating. Such oscillation is not desirable in micropositioning because the overshoot can cause unwanted unstability of the positioned objects.
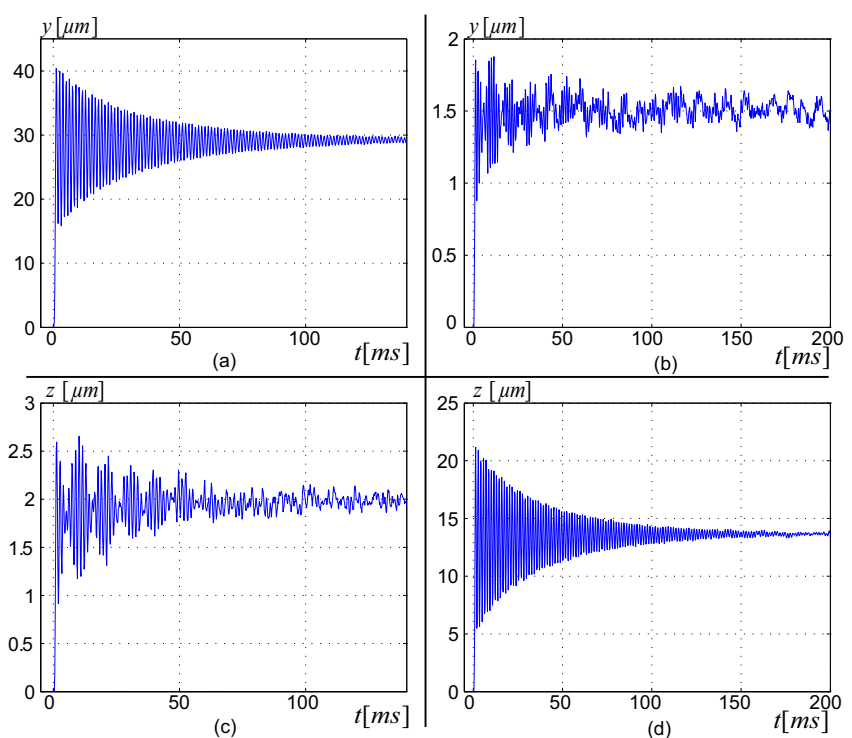

Fig. 3. Step responses of the piezocantilever.

Each axis $y$ and $z$ of the piezocantilever is characterized by a hysteresis nonlinearity. On the other hand, the actuator undergoes undesirable couplings: when a voltage $U_{y}$ (resp. $U_{z}$ ) is applied to the cantilever, the axis $z$ (resp. $y$ ) also bends. The 2-DOF cantilever can therefore be seen as a bi-input-bi-output (BIBO) nonlinear system (Fig. 4). In the following, we propose first to linearize it by applying a feedforward controller to compensate the hysteresis. Afterwards, a linear feedback controller is proposed.

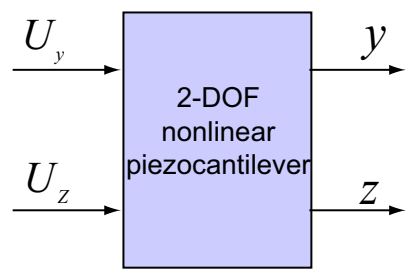

Fig. 4. The 2-DOF piezocantilever: a nonlinear BIBO system.

\section{HYSTERESIS COMPENSATION}

The compensation of hysteresis in piezoelectric materials have attracted many attention. Several approaches have been proposed for that but the Prandtl-Ishlinskii (PI) one is recognized for its accuracy, ease of computation and implementation allowing it very convenient for real-time applications [12][13]. This approach will be used in our application.

\section{A. Modeling and parameters identification}

In the sequel, the definitions and equations are given for only $y$, but they also hold for $z$. A PI hysteresis model is based on the sum of elementary hysteresis called playoperator. A play operator is defined by:

$$
\left\{\begin{array}{l}
y(t)=\max \left\{U_{y}(t)-r, \min \left\{U_{y}(t)+r, y(t-T)\right\}\right\} \\
y(0)=y_{0}
\end{array}\right.
$$

where $T$ is the sampling time and $r$ the radius of the play-operator.

So, a hysteresis modeled by the PI approach is given by:

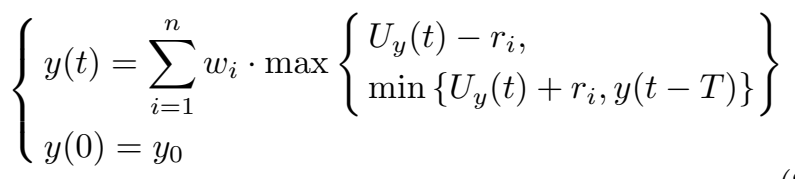

where $n$ is the number of play-operators and $w_{i}$ the weighting. If $n$ is high, the model is more precise but may become complex.

The identification of the parameters $w_{i}$ and $r_{i}$, well detailed in [13], is performed by using a sine input voltage $U_{y}\left(U_{z}\right.$ for $z$-axis $)$. The amplitude of the sine $\left(U_{y}=10 \mathrm{~V}\right)$ is chosen to cover the expected range of deflection. After different trial, a number $n=15$ for each model ( $y$ and $z$ ) is a good compromise. Fig. 5 give a comparison of the experimental hysteresis and the identified models which show that they well fit.
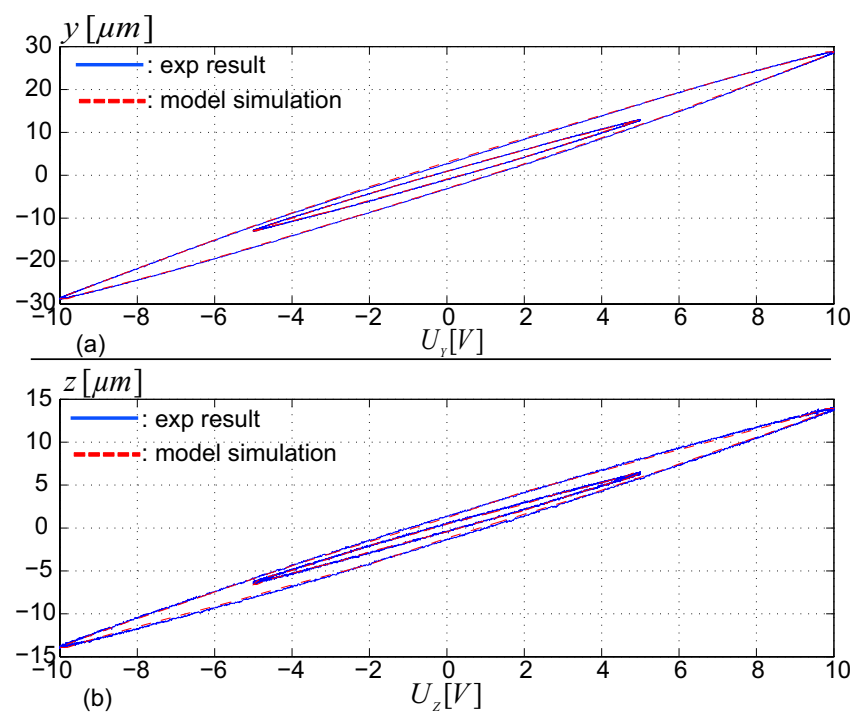

Fig. 5. Hysteresis characteristics: experimental results and models simulation.

\section{B. Compensation}

To compensate a hysteresis approximated by the PI model, another PI-model is used as compensator. This compensator has as input a reference $y_{h c}$ (resp. $z_{h c}$ ) and as output the control votage $U_{y}$ (resp. $U_{z}$ ). The principle of compensation is such that the two hysteresis are symmetrical relative to the straightline with slope equal to unity. If the compensator is denoted by: 


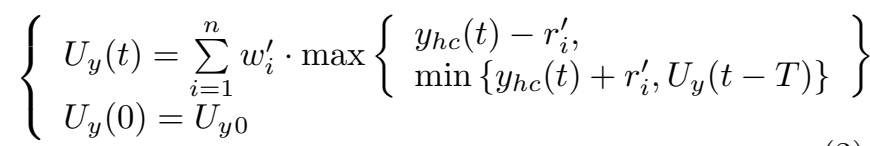

thus, the weighting $w_{i}^{\prime}$ and the radius $r_{i}^{\prime}$ are computed as follows [19]:

$$
r_{k}^{\prime}=\sum_{j=1}^{k} w_{j} \cdot\left(r_{k}-r_{j}\right) \quad ; \quad k=1 \cdots n
$$

and

$$
\begin{aligned}
& w_{1}^{\prime}=\frac{1}{w_{1}} \\
& w_{k}^{\prime}=\frac{-w_{k}}{\left(w_{1}+\sum_{j=2}^{k} w_{j}\right) \cdot\left(w_{1}+\sum_{j=2}^{k-1} w_{j}\right)} \quad ; \quad k=2 \cdots n
\end{aligned}
$$

Using the calculation in (Eq.4) and (Eq.5), the two compensators (for $y$ and for $z$ ) were computed and implemented. Fig. 6 presents the block-scheme of the compensators and of the piezoactuator.

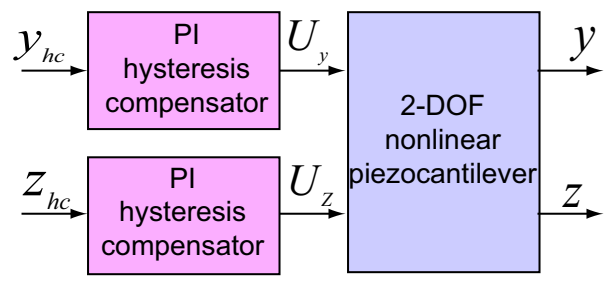

Fig. 6. Block scheme of the feedforward control of the hysteresis.

To characterize the efficiency of the two compensators, a sine reference input $y_{h c}$ is first applied. Then, the resulting outputs $y$ and $z$ are recorded and plotted w.r.t. $y_{h c}$ (see Fig. 7-a and c respectively). Afterwards, a sine reference $z_{h c}$ is applied and the corresponding outputs $y$ and $z$ are also plotted (see Fig. 7-b and d respectively). As we can see in the figures, the initial hysteresis for the two axis are clearly reduced (Fig. 7-a and d) which enables us to consider the system as linear. However, the system still undergoes coupling because the influence of $y_{h c}$ (resp. $z_{h c}$ ) on $z$ (resp. $y$ ) still remains (Fig. 7-b and c respectively). In the next section, a feedback controller is proposed to remove this coupling. The controller also ensures that all external disturbance will be rejected.

\section{IMC CONTROL}

In this section, we synthesize a feedback controller for the 2-DOF piezocantilever with hysteresis compensators presented in Fig. 6. An IMC (internal model control) scheme is proposed. Its main advantages are the ease of computation and the low order of the controller. Furthermore, there is no requirement to have a model of the coupling. First, we model and identify the new system to be controlled (piezocantilever + hysteresis compensators).
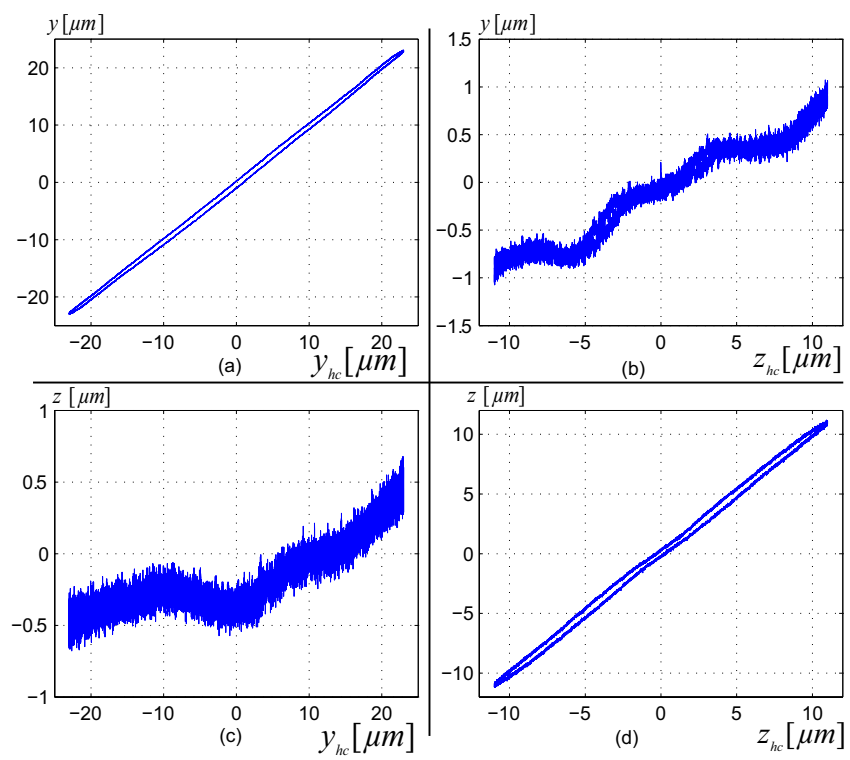

Fig. 7. Static characteristics of the linearized system.

\section{A. Modeling}

From the results in Fig. 7, it can be deduced that the model of the compensated system in Fig. 6 is BIBO and linear. In [18], it has been demonstrated that couplings are additive relative to output signals for piezocantilevers. Taking into account that result, we derive the model of our system:

$$
\left\{\begin{array}{l}
y=K_{y} D_{y}(s) y_{h c}+c_{y}+d_{e x t-y} \\
z=K_{z} D_{z}(s) z_{h c}+c_{z}+d_{e x t-z}
\end{array}\right.
$$

where $K_{i}(i \in\{y, z\})$ are the static gains, $D_{i}(s)$ are the dynamic part such as $D_{i}(0)=1, c_{y}$ (resp. $c_{z}$ ) is the coupling due to $z_{h c}$ (resp. $y_{h c}$ ), and $d_{e x t-i}$ are the eventual external disturbances. The couplings $c_{y}$ and $c_{z}$ can be precisely modeled using Fig. 7-b and c respectively, however we propose to consider them as also external disturbances. The objective is that only the linear characteristic that corresponds to the direct transfers (see Fig. 7-a and d) has to be modeled and no characterization of the couplings is performed. So, we assemble the coupling $c_{i}$ with the external $d_{e x t-i}$ such that $d_{i}=c_{i}+d_{e x t-i}$. Thus, the final model is:

$$
\left\{\begin{array}{l}
y=K_{y} D_{y}(s) y_{h c}+d_{y} \\
z=K_{z} D_{z}(s) z_{h c}+d_{z}
\end{array}\right.
$$

\section{B. Identification}

The identification of the static gains $K_{y}$ and $K_{z}$ are straightforward by calculating the slopes $\frac{\partial y}{\partial y_{h c}}$ and $\frac{\partial z}{\partial z_{h c}}$ respectively from Fig. 7-a and d. We have: $K_{y}=K_{z}=1$.

The identification of the dynamics $D_{y}(s)$ and $D_{z}(s)$ is be performed by using step responses of the system to be controlled, i.e. of the piezocantilever with hysteresis compensators. However, the used PI hysteresis compensators being static and rate-independant, they are not 
affected by and conversely do not affect the dynamics of piezoactuators [13]. The dynamics $D_{y}(s)$ and $D_{z}(s)$ can therefore be identified using the step responses of the piezocantilever without hysteresis compensators which are pictured in Fig. 3-a and d. Applying systems identification [20] and Matlab, we obtain:

$$
\left\{\begin{array}{l}
D_{y}=\frac{-0.015(s+7461)(s-3601)}{(s+2328)(s+172)} \\
D_{z}=\frac{0.0107\left(s^{2}+8997 s+3 \times 10^{7}\right)}{(s+1992)(s+165)}
\end{array}\right.
$$

\section{Controller synthesis}

If $C_{y}(s)$ and $C_{z}(s)$ denote the controller for $y$ and $z$ axis respectively, the bloc-scheme of the closed-loop which is based on the IMC-scheme is given in Fig. 8. In this, a model $\hat{G}_{i}(s)=K_{i} D_{i}(s)(i \in\{y, z\})$ is put in parallel with the real process. Signals $d_{i}$ are the previously defined disturbances and $y_{r}$ and $z_{r}$ are the reference points.

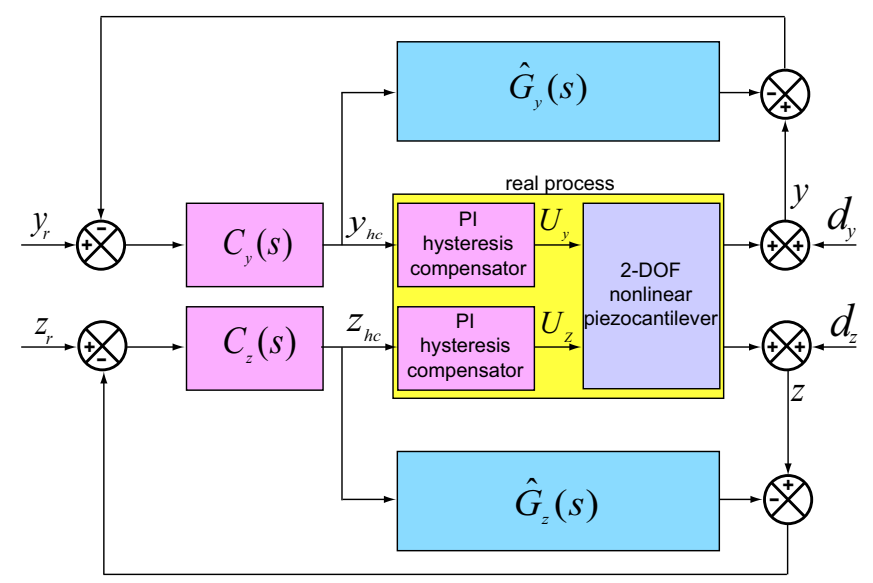

Fig. 8. Closed-loop scheme of the 2-DOF piezocantilever.

Denote $G_{y}(s)$ and $G_{z}(s)$ the real models for each axis. Thus, $\left(G_{y}-\hat{G}_{y}\right)$ and $\left(G_{z}-\hat{G}_{z}\right)$ quantify the model errors (uncertainty). From Fig. 8, we derive the equations of the outputs:

$$
\left\{\begin{array}{l}
y=\frac{C_{y} G_{y}}{\left(1+C_{y}\left(G_{y}-\hat{G}_{y}\right)\right)} y_{r}+\frac{\left(1-C_{y} \hat{G}_{y}\right)}{\left(1+C_{y}\left(G_{y}-\hat{G}_{y}\right)\right)} d_{y} \\
z=\frac{C_{z} G_{z}}{\left(1+C_{z}\left(G_{z}-\hat{G}_{z}\right)\right)} z_{r}+\frac{\left(1-C_{z} \hat{G}_{z}\right)}{\left(1+C_{z}\left(G_{z}-\hat{G}_{z}\right)\right)} d_{z}
\end{array}\right.
$$

From (Eq.9), we can see that if $C_{i}(s)=\frac{1}{\hat{G}_{i}(0)}=$ $\frac{1}{K_{i}}=1(i \in\{y, z\})$ and if $\hat{G}_{i}(s)=G_{i}(s)$ (i.e. perfect models are used), then perfect constant setpoint tracking and constant disturbance rejection is achieved. However, even if $\hat{G}_{i}(s) \neq G_{i}(s)$, perfect disturbance rejection is still realised provided $C_{i}(s)=\frac{1}{\hat{G}_{i}(0)}$. To improve the robustness by minimizing the effects of model errors which usually occur at high frequency, a low-pass filter $F_{i}(s)$ is introduced and the controllers become: $C_{y}(s)=$ $\frac{1}{\hat{G}_{y}(0)} F_{y}(s)=F_{y}(s)$ and $C_{z}(s)=\frac{1}{\hat{G}_{z}(0)} F_{z}(s)=F_{z}(s)$. The order of the filter is chosen such that the controller is proper. Furthermore, the behavior of the closed-loop can be imposed by this filter. In our case, we choose a first-order behavior with a settling time of $20 \mathrm{~ms}$ for the closed-loop both in $y$-axis and $z$-axis. These specifications yields $F_{y}(s)=F_{z}(s)=\frac{1}{1+\frac{20}{3} s}$. Finally, we have:

$$
C_{y}=\frac{1}{1+\frac{20}{3} s} \quad \text { and } \quad C_{z}=\frac{1}{1+\frac{20}{3} s}
$$

As we can see, the controllers have a very low order, contrary to that proposed in [18] which yields controllers with orders up to 4.

\section{Experimental results}

The calculated controllers $C_{i}(s)$ as well as the models $\hat{G}_{i}(s)$ were implemented in Matlab-Simulink following the scheme in Fig. 8. The first experiment consists in analyzing the step response and evaluating the general performances. For that, we apply first a step reference input $y_{r}=23 \mu \mathrm{m}$ while $z_{r}=$ is left equal to zero. As we can see in Fig. 9-a, the statical error is null and the settling time is nearly $20 \mathrm{~ms}$. The effect of $y_{r}$ to $z$-axis is pictured in Fig. 9-c. It demonstrates that the coupling is negligible $\left(\leq \frac{0.3 \mu \mathrm{m}}{23 \mu \mathrm{m}}\right)$ and quickly rejected. Afterwards, a step reference input $z_{r}=11 \mu \mathrm{m}$ is applied and $y_{r}$ is set to zero. Fig. 9-d shows that the statical error is null and the settling time is also respected $(\approx 20 \mathrm{~ms})$. In Fig. $9-\mathrm{b}$, it is shown that the coupling $\approx \frac{0.5 \mu m}{11 \mu m}$ is also rejected. These step responses (Fig. 9-a and d) show that the behaviors of the closed-loop follow that of a first-order system as required in the specifications.
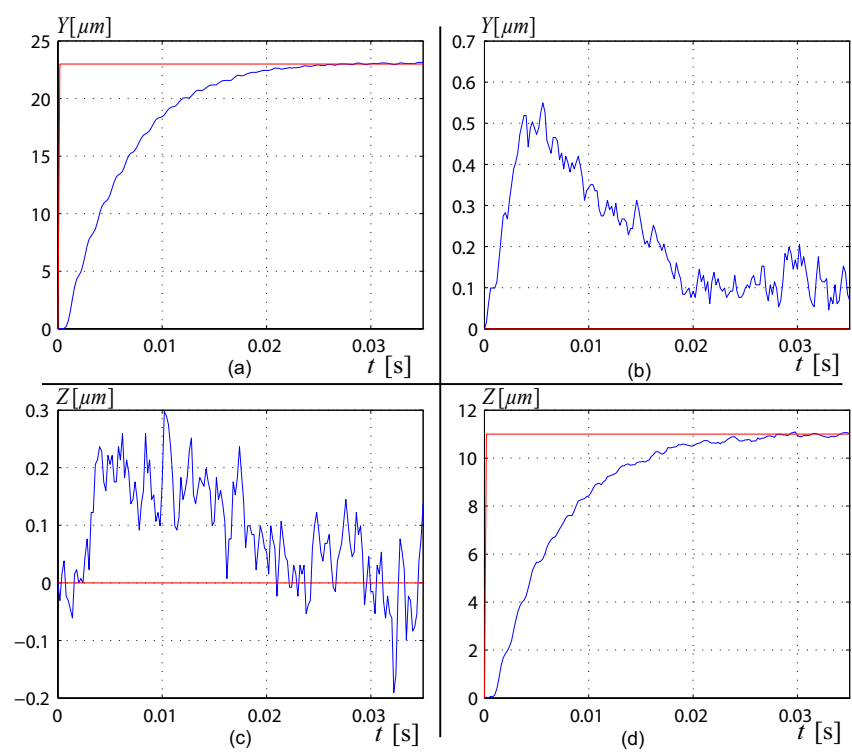

Fig. 9. Step responses of the closed-loop.

The next experiment consists in applying a series of steps. The results are pictured in Fig. 10. First, we remark that the couplings are completely negligible face to the different steps since they are confused with the noises of the sensors (Fig. 10-b and c). We also appreciate the closed-loop piezocantilever which well tracks these step 
inputs. These results confirm the efficiency of the IMCfeedback control scheme combined with the hysteresis feedforward control as proposed in this paper.
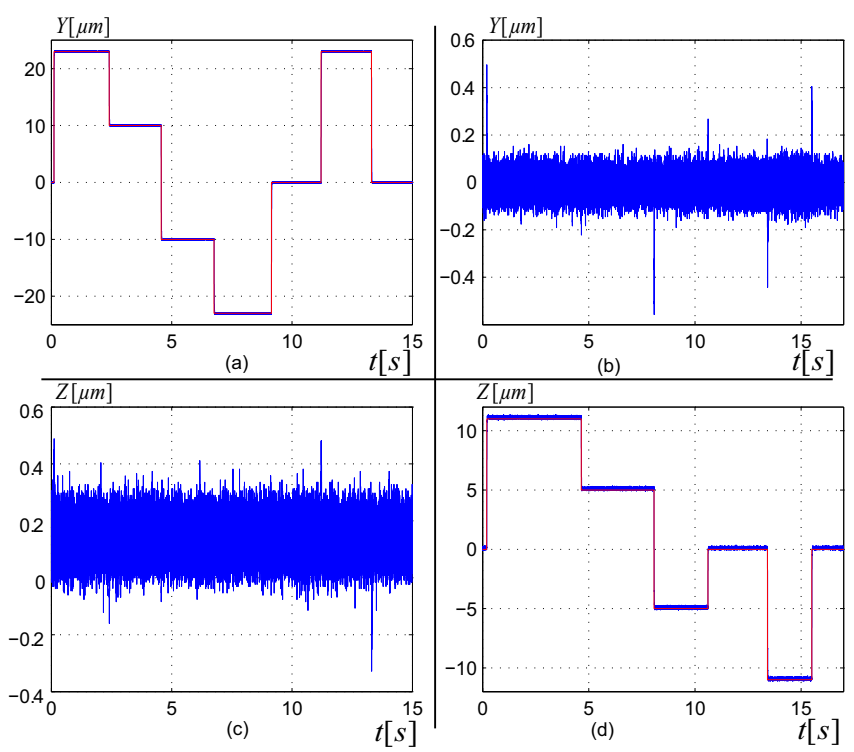

Fig. 10. Responses of the closed-loop to a series of steps.

\section{CONClusion}

This paper presented the control of a 2-DOF piezocantilever dedicated to precise and further automated positioning. The piezocantilever exhibits hysteresis nonlinarity and strong coupling between the two axis which make lose its accuracy. Furthermore, these characteristics also make difficult the synthesis of linear, simple and embeddable controllers. We therefore proposed to first linearize the system by feedforward controlling the hysteresis. The Prandtl-Ishlinskii approach is used because of its conveniency to real-time applications and to its accuracy. Afterwards, we apply a feedback controller in order to enhance the performances and to reject the coupling between the axis as well as eventual external disturbances. The internal model control (IMC) scheme is used for the feedback. Its main advantages are the robustness relative eventual model uncertainty, the ease of computation of the controller and its loworder. The experimental results confirm the efficiency of the proposed approach to control the nonlinear 2-DOF piezocantilever.

\section{ACKNOWLEDGMENT}

This work is supported by the French research project NANOROL (ANR $N^{\circ}$ PSIROB07_184846).

\section{REFERENCES}

[1] A. Bergander, W. Driesen, T. Varidel, M. Meizoso and J. M. Breguet, "Mobile $\mathrm{cm} 3$-microrobots with tools for nanoscale imaging and micromanipulation" MechRob 2004, pp.1041-1047, 13-15 Aachen, Germany, Sept. 2004.
[2] S. Fatikow, T. Wich, H. Hülsen, T. Sievers and M. Jähnisch, "Microrobot system for automatic nanohandling inside a scanning electron microscope", IEEE/ASME Transactions on Mechatronics, 12(3), pp.244-252, 2007.

[3] Micky Rakotondrabe, Yassine Haddab and Philippe Lutz, "Development, Modeling, and Control of a Micro-/Nanopositioning 2-DOF Stick-Slip Device", IEEE - Transactions on Mechatronics (T-mech), Vol.14, Issue 6, pp:733-745, December 2009.

[4] http://www.physikinstrumente.com/

[5] http://www.piezotechnologies.com/

[6] Micky Rakotondrabe and Alexandru Ivan, "Development and dynamic modeling of a new hybrid thermo-piezoelectric microactuator", IEEE - Transactions on Robotics (T-RO), Vol.26, Issue.6, pp.1077-1085, December 2010.

[7] Y. Haddab, N. Chaillet and A. Bourjault, 'A microgripper using smart piezoelectric actuators', IEEE/RSJ IROS, Takamatsu Japan, October-November 2000.

[8] D. J. Capparelli, M. I. Frecker, T. W. Simpson and A. Snyder, 'Design of a PZT bimorph actuator using a metamodel-based approach', Transactions of the ASME, Journal of Mechanical Design, pp.354-357, 2002

[9] H. Xinhan, C. Jianhua, W. Min and L. Xiadong , 'A piezoelectric bimorph micro-gripper with micro-force sensing', IEEE ICIA, June-July 2005.

[10] J. Agnus, P. Nectoux and N. Chaillet, 'Overview of microgrippers and design of a micro-manipulation station based on a MMOC microgripper', IEEE CIRA, Espoo Finland, june 2005.

[11] D. Croft, G. Shed and S. Devasia, "Creep, hysteresis and vibration compensation for piezoactuators: atomic force microscopy application", ASME Journal of Dynamic Systems, Measurement and Control, 2001.

[12] B. Mokaberi and A. A. G. Requicha, "Compensation of scanner creep and hysteresis for AFM nanomanipulation", IEEE Transactions on Automation Science and Engineering, Vol.5, $\mathrm{N}^{\circ} 2$, pp.197-208, 2008.

[13] Micky Rakotondrabe, Cédric Clévy and Philippe Lutz, "Complete open loop control of hysteretic, creeped and oscillating piezoelectric cantilever", IEEE - Transactions on Automation Science and Engineering, Vol.7(3), pp:440-450, July 2010.

[14] Micky Rakotondrabe, Yassine Haddab and Philippe Lutz, 'Quadrilateral modelling and robust control of a nonlinear piezoelectric cantilever', IEEE - Transactions on Control Systems Technology, Vol.17, Issue 3, pp:528-539, May 2009.

[15] K. K. Leang and S. Devasia, "Feedback-linearized inverse feedforward for creep, hysteresis, and vibration compensation in piezoactuators", IEEE Control Systems Technology: Special Issue on Dynamics and Control of Micro- and Nano-Scale Systems, Vol 15, No. 5, 927 - 935, 2007.

[16] P. de Lit, J. Agnus, C. Clévy and N. Chaillet, "A four-degreeof-freedom microprehensile microrobot on chip", International Journal of Assembly Technology and Management (Assembly Automation), 2004

[17] Zhou, Q., Korhonen, P., Laitinen, J., Sjövall, S. "Automatic dextrous microhandling based on a 6 DOF microgripper", Journal of Micromechatronics, Vol. 3, No. 3-4, pp. 359-387, 2006.

[18] Micky Rakotondrabe, Kanty Rabenorosoa, Joël Agnus and Nicolas Chaillet, "Robust feedforward-feedback control of a nonlinear and oscillating 2-dof piezocantilever", IEEE - Transactions on Automation Science and Engineering (T-ASE), DOI.10.1109/TASE.2010.2099218.

[19] K. Kuhnen and H. Janocha, "Inverse feedforward controller for complex hysteretic nonlinearities in smart-materials systems", Control of Intelligent System, Vol.29(3), pp.74-83, 2001.

[20] L. Ljung, "System identfification toolbox", The Matlab user's guide. 